\title{
Sensitivity of the Polyakov loop and related observables to chiral symmetry restoration
}

\author{
D. A. Clarke $\odot,{ }^{1}$ O. Kaczmarek $\odot,{ }^{2,1}$ F. Karsch, ${ }^{1}$ Anirban Lahiri, ${ }^{1}$ and Mugdha Sarkar ${ }^{1}$ \\ ${ }^{1}$ Fakultät für Physik, Universität Bielefeld, D-33615 Bielefeld, Germany \\ ${ }^{2}$ Key Laboratory of Quark \& Lepton Physics (MOE) and Institute of Particle Physics, \\ Central China Normal University, Wuhan 430079, China
}

(Received 31 August 2020; accepted 7 January 2021; published 29 January 2021; corrected 30 March 2021)

\begin{abstract}
While the Polyakov loop is an order parameter of the deconfinement transition in the heavy quark mass regime of QCD, its sensitivity to the deconfinement of light, dynamical quarks in QCD is not apparent. On the other hand, the quark mass dependence of the Polyakov loop is sensitive to the appearance of a chiral phase transition. Using lattice QCD calculations in the staggered fermion discretization scheme at finite values of the lattice spacing, $a T=1 / 8$, we show here, for the first time, that the Polyakov loop expectation value, and the heavy quark free energy extracted from it, behaves like an energylike observable in the vicinity of the chiral phase transition temperature $T_{c}$. Consistent with scaling behavior of energylike observables in the $3 d, \mathrm{O}(2)$ universality class, the quark mass derivatives diverge in the chiral limit at $T_{c}$ while the temperature derivatives stay finite. The latter will develop a characteristic spike at $T_{c}$. This, however, may be resolved only in calculations with quark masses being 2 orders of magnitude smaller than those currently accessible in lattice QCD calculations.
\end{abstract}

DOI: 10.1103/PhysRevD.103.L011501

\section{INTRODUCTION}

The Lagrangian of quantum chromodynamics (QCD), the theory describing interactions controlled by the strong force, possesses exact global symmetries only in the massless (chiral) and infinite quark mass (pure gauge) limits. The latter case has been extensively exploited in lattice QCD calculations to discuss the deconfinement phase transition in pure gauge theories [1-3] and its imprint in the heavy quark sector of QCD. Also, in the light quark mass region, the rapid change of the Polyakov loop expectation value, $\langle P\rangle$, as function of the temperature, characterized by an inflection point in its $T$-dependence, is often taken as an indication for the occurrence of deconfinement. However, studies with (almost) physical light up and down quark masses and improved discretization schemes for the QCD Lagrangian, performed closer to the continuum limit, in general show that the QCD transition is a smooth crossover, and no evidence for an inflection point in the vicinity of the chiral transition temperature is found [4-7].

In the limit of vanishing values of the two light quark masses, the chiral flavor symmetry, $\mathrm{SU}(2)_{L} \times \mathrm{SU}(2)_{R}$, gets

Published by the American Physical Society under the terms of the Creative Commons Attribution 4.0 International license. Further distribution of this work must maintain attribution to the author(s) and the published article's title, journal citation, and DOI. Funded by SCOAP ${ }^{3}$. restored above a temperature $T_{c}$, giving rise to a chiral phase transition [8]. In QCD, the nonzero light quark masses, $m_{l}$, are small on the scale of relevant temperatures, e.g., $T_{c}$. Thus, the light quark chiral condensate, $\langle\bar{\psi} \psi\rangle$, is a good indicator for the occurrence of a phase transition in the chiral limit of QCD. The maxima in either the quark mass or temperature derivatives of $\langle\bar{\psi} \psi\rangle$ diverge in the chiral limit, and for $m_{l}>0$ the positions of these maxima define pseudocritical temperatures that converge to $T_{c}$ in the chiral limit.

The Polyakov loop is a purely gluonic observable that is trivially invariant under chiral transformations in the fermion sector of the QCD Lagrangian. As far as critical behavior close to a second order phase transition point is concerned, it thus may be expected that $\langle P\rangle$ as well as the heavy quark free energy, $F_{q}=-T \ln \langle P\rangle$, behave like any other "energylike" operator that may appear in an effective Hamiltonian describing e.g., QCD thermodynamics in the vicinity of the chiral phase transition. One may thus expect that $\langle P\rangle$ as well as $F_{q} / T$ are sensitive to critical behavior arising from this transition.

Here we present results on the temperature and quark mass dependence of $\langle P\rangle$ and $F_{q} / T$ close to the chiral limit. We show that both of them reflect properties of energylike observables in the vicinity of $T_{c}$ and discuss the resulting chiral limit behavior. As we will perform calculations at a nonzero value of the lattice spacing, using the highly improved staggered quark (HISQ) action, the relevant symmetry group for the discussion of universal scaling 
properties is the $\mathrm{O}(2)$ rather than $\mathrm{O}(4)$ group as it will be the case in the continuum limit of lattice QCD. However, as will become clear in the following, none of the qualitative features that will arise from the energylike behavior of the Polyakov loop in theories with global $\mathrm{O}(N)$ symmetry will depend on this difference.

\section{POLYAKOV LOOP AND THE HEAVY QUARK FREE ENERGY}

For lattice QCD in a finite Euclidean space-time volume $N_{\sigma}^{3} \times N_{\tau}$, the Polyakov loop, $P_{\vec{x}}$, and its spatial average, $P$,

$$
P_{\vec{x}}^{\text {bare }} \equiv \frac{1}{3} \operatorname{tr} \prod_{\tau} U_{4}(\vec{x}, \tau), \quad P \equiv \frac{1}{N_{\sigma}^{3}} \mathrm{e}^{N_{\tau} c\left(g^{2}\right)} \sum_{\vec{x}} P_{\vec{x}}^{\text {bare }},
$$

are given in terms of $\mathrm{SU}(3)$-valued field variables, $U_{4}(\vec{x}, \tau)$, defined on the temporal link $(\mu=4)$ originating at a Euclidean space-time point $(\vec{x}, \tau)$. The volume $V=$ $\left(N_{\sigma} a\right)^{3}$ and inverse temperature $T^{-1}=N_{\tau} a$ are given in terms of the lattice spacing $a$. The bare Polyakov loop, $P^{\text {bare }}$, has been renormalized using renormalization constants, $c\left(g^{2}\right)$, determined in Ref. [6] (Table V) for the regularization scheme used also in this work.

The heavy quark free energy, $F_{q} / T$, characterizes the behavior of correlation functions between static quark and antiquark sources at infinite distances [9],

$$
F_{q}(T, H)=-T \ln \langle P\rangle=-\frac{T}{2} \lim _{|\vec{x}-\vec{y}| \rightarrow \infty} \ln \left\langle P_{\vec{x}} P_{\vec{y}}^{\dagger}\right\rangle .
$$

Here $H=m_{l} / m_{s}$ parametrizes the quark mass dependence in terms of the ratio of degenerate light quark masses $m_{l} \equiv m_{u}=m_{d}$ and the strange quark mass $m_{s}$. For the analysis of the quark mass dependence of the heavy quark free energy, we calculate the renormalization-schemeindependent mixed susceptibility,

$$
\frac{\partial F_{q}(T, H) / T}{\partial H}=-\frac{1}{\langle P\rangle} \frac{\partial\langle P\rangle}{\partial H} \equiv-\frac{\chi_{m P}}{\langle P\rangle},
$$

with the quark mass derivative of $\langle P\rangle$ given by

$$
\chi_{m P} \equiv \frac{\partial\langle P\rangle}{\partial H}=\langle P \cdot \Psi\rangle-\langle P\rangle\langle\Psi\rangle .
$$

Here $\Psi \equiv \frac{1}{2} \hat{m}_{s} \operatorname{tr} M_{l}^{-1}$ denotes the extensive observable defining a dimensionless combination of the two-flavor light quark chiral condensate in terms of the light quark, staggered fermion matrix $M_{l} ; \hat{m}_{s}$ is the bare strange quark mass in lattice units, $m_{s} / T=\hat{m}_{s} N_{\tau}$.

Using fit results for $F_{q} / T$ and its derivative with respect to the quark mass, we will also be able to determine the derivatives of $\langle P\rangle$ and $F_{q} / T$ with respect to $T$. Similar to the $\mathrm{H}$-derivatives, these derivatives are closely related to each other,

$$
T_{c} \frac{\partial F_{q}(T, H) / T}{\partial T}=-\frac{T_{c}}{\langle P\rangle} \frac{\partial\langle P\rangle}{\partial T} .
$$

\section{POLYAKOV LOOP AND CHIRAL SYMMETRY RESTORATION}

Within Wilson's renormalization group approach [10,11], thermodynamics in the vicinity of a critical point can be described by an effective Hamiltonian, which is defined in a multidimensional space of operators (observables). These operators may be invariant under the global symmetry that gets broken at the critical point or may break this symmetry explicitly. In the former case, the operator is said to be energylike, while in the latter case it is magnetizationlike. In QCD the two-flavor, light quark chiral condensate is a typical magnetizationlike operator.

The Polyakov loop is invariant under chiral transformations of the quark fields. Its expectation value, $\langle P\rangle$, as well as the heavy quark free energy, $F_{q} / T$, thus are energylike observables. We expect that they are sensitive to the chiral phase transition to the extent that they receive nonanalytic (singular) contributions in addition to analytic (regular) terms. In the vicinity of the critical point, i.e., close to $(T, H)=\left(T_{c}, 0\right)$, nonanalytic contributions are universal scaling functions of a scaling variable $z=z_{0} t H^{-1 / \beta \delta}$ with $t=\left(T-T_{c}\right) / T_{c}$ and $z_{0}, T_{c}$ being nonuniversal constants. Energylike observables receive contributions from the scaling function, $f_{f}^{\prime}(z)=\mathrm{d} f_{f}(z) / \mathrm{d} z$, of the $3 d, \mathrm{O}(N)$ universality class [12], which is the derivative of the scaling function $f_{f}(z)$ that characterizes the singular part of the logarithm of the partition function. For the heavy quark free energy, we use the scaling ansatz,

$$
F_{q}(T, H) / T=A H^{(1-\alpha) / \beta \delta} f_{f}^{\prime}(z)+f_{\mathrm{reg}}(T, H),
$$

where $A$ is another nonuniversal constant and the critical exponents are $\beta, \delta$, and $\alpha=2-\beta(1+\delta)$. The regular contribution is an analytic function, which close to $\left(T_{c}, 0\right)$ can be given as a Taylor series with even powers in $H$,

$$
f_{\text {reg }}(T, H)=\sum_{i, j} a_{i, 2 j}^{r} t^{i} H^{2 j} \equiv \sum_{j} p_{2 j}^{r}(T) H^{2 j} .
$$

Using Eqs. (1) and (5), the Polyakov loop expectation value may be written as

$$
\langle P\rangle_{T, H}=\exp \left(-A H^{(1-\alpha) / \beta \delta} f_{f}^{\prime}(z)-f_{\text {reg }}(T, H)\right) .
$$

Note that for $(T, H)$ close to $\left(T_{c}, 0\right)$ Eq. (7) reduces to the usual nonexponential scaling ansatz. 
Using the known asymptotic behavior of $f_{f}(z)$ for $z \rightarrow \pm \infty$ [12], we obtain from Eq. (5) for the quark mass dependence of $F_{q} / T$ at fixed $T$ and for small $H$,

$$
\frac{F_{q}(T, H)}{T} \sim \begin{cases}a^{-}(T)+A p_{s}^{-}(T) H, & T<T_{c} \\ a_{0,0}^{r}+A a_{1} H^{(1-\alpha) / \beta \delta}, & T=T_{c}, \\ a^{+}(T)+p^{+}(T) H^{2}, & T>T_{c},\end{cases}
$$

with $a^{ \pm}(T)=A a_{s}^{ \pm}(T)+f_{\text {reg }}(T, 0)$ as well as $p^{+}(T)=$ $A p_{s}^{+}(T)+p_{2}^{r}(T)$ receiving contributions from both the singular and regular terms. For $T \leq T_{c}$, the dominant quark mass dependence arises from the singular term only. In particular, we have

$$
\begin{aligned}
& a_{s}^{ \pm}(T)=(2-\alpha) z_{0}^{1-\alpha} c_{0}^{ \pm} t|t|^{-\alpha}, \\
& p_{s}^{-}(T)=(2-\alpha-\beta \delta)\left(-z_{0} t\right)^{1-\alpha-\beta \delta}, \\
& p_{s}^{+}(T)=(2-\alpha-2 \beta \delta) c_{1}^{+}\left(z_{0} t\right)^{1-\alpha-2 \beta \delta},
\end{aligned}
$$

where we followed the notation of Ref. [12] with $c_{0}^{ \pm}, c_{1}^{+}$, and $a_{1}$ denoting coefficients appearing in the parametrization of the scaling function $f_{f}(z)$.

For the $3 d, \mathrm{O}(4)$ universality class these coefficients are given in [12]. As we will present here only, results from calculations for one nonzero value of the lattice spacing, $a=1 / 8 T$, and do not perform a continuum extrapolation, we will use the critical exponents and scaling functions of the $3 d, \mathrm{O}(2)$ universality class. We extracted these from [13], where the scaling functions are parametrized in the Widom-Griffiths form. We use $a_{1}=0.4734, c_{0}^{-}=2.447$, $c_{0}^{+}=2.728, c_{1}^{+}=-0.678$, along with $\beta=0.349, \delta=$ 4.780 [13-15].

Making use of the relation between scaling functions, $f_{f}(z)$ and that of the order parameter, $f_{G}(z)$ (see e.g., [12]), we obtain in the vicinity of $T_{c}$,

$\frac{\partial F_{q}(T, H) / T}{\partial H}=-A H^{(\beta-1) / \beta \delta} f_{G}^{\prime}(z)+\frac{\partial f_{\mathrm{reg}}(T, H)}{\partial H}$.

The derivative of $F_{q} / T$ with respect to $T$ is given by

$T_{c} \frac{\partial F_{q}(T, H) / T}{\partial T}=A z_{0} H^{-\alpha / \beta \delta} f_{f}^{\prime \prime}(z)+T_{c} \frac{\partial f_{\mathrm{reg}}(T, H)}{\partial T}$.

As $\alpha / \beta \delta<0$ for the $3 d, \mathrm{O}(2)$ as well as $\mathrm{O}(4)$ universality classes, the derivatives of $\langle P\rangle$ and $F_{q} / T$ with respect to $T$ do not diverge at $T_{c}$ in the limit $H \rightarrow 0$. On the other hand, the corresponding quark mass derivatives, Eq. (10), are expected to diverge when approaching the chiral limit at $T_{c}$ as well as when approaching $T_{c}$ from below at $H=0$. A possible influence of the chiral phase transition on the quark mass dependence of $\langle P\rangle$ and $F_{q} / T$ is thus much easier to establish than on their temperature dependence.

\section{COMPUTATIONAL SETUP AND DATA ANALYSIS}

In this study, we analyze properties of $(2+1)$-flavor QCD where the strange quark mass has been kept fixed to its physical value and the two degenerate light quark masses are varied in the range $H=1 / 20$ to $1 / 160$, corresponding to a pion mass $160 \mathrm{MeV} \gtrsim m_{\pi} \gtrsim 58 \mathrm{MeV}$ [16]. The analysis is performed on sets of gauge field configurations that had been generated previously by the HotQCD Collaboration [16-19] on lattices of size $32^{3} \times 8 \quad(H=1 / 20,1 / 27), 40^{3} \times 8 \quad(H=1 / 40), \quad$ and $56^{3} \times 8(H=1 / 80,1 / 160)$ using HISQ [20] and the tree-level improved Symanzik gauge action. These data have previously been used in calculations of pseudocritical temperatures [21] and the chiral phase transition temperature of $(2+1)$-flavor QCD [16]. We also have generated additional configurations for $H=1 / 40,1 / 80$. For the scale setting, we use the kaon decay constant obtained in calculations with the HISQ action, i.e., $f_{K}=$ 156.1/ $\sqrt{2} \mathrm{MeV}$ [22].

Observables have been calculated on lattices with temporal extent $N_{\tau}=8$ and $N_{\sigma} / N_{\tau}=4-7$. For $H=1 / 80$, we have results for the entire range of aspect ratios, $N_{\sigma} / N_{\tau}$. An analysis of finite volume effects on $\langle P\rangle$ shows no significant volume dependence of $\langle P\rangle$ in the entire temperature range. Results for different $N_{\sigma} / N_{\tau}$ agree within errors of about $1 \%$. We thus can safely neglect any finite volume corrections to our results for $\langle P\rangle$. The statistics used in this study can be found in [15], along with more details for the finite volume dependence of $\langle P\rangle$.

\section{RESULTS}

In Fig. 1 (left), we show results for the derivative of $F_{q}(T, H) / T$ with respect to $H$ as function of $T$. It is apparent that $\partial\left(F_{q} / T\right) / \partial H$ decreases with quark mass at high temperature and increases at low temperature. This is consistent with an approach toward zero at high $T$ and a nonvanishing, strongly temperature dependent constant at low $T$. Such a pattern is in accordance with the expected quadratic dependence on $H$ for $T>T_{c}$ and the linear dependence for $T<T_{c}$ given in Eq. (8). Although errors are large for the results obtained with the smallest light quark mass, $H=1 / 160$, it is evident that $\partial\left(F_{q} / T\right) / \partial H$ has maxima at $T \sim 145-150 \mathrm{MeV}$ which are close to the chiral phase transition temperature on lattices with temporal extent $N_{\tau}=8$ determined in [16], $T_{c}^{N_{\tau}=8}=144(2) \mathrm{MeV}$. With decreasing $H$, they approach $T_{c}^{N_{\tau}=8}$ and the peak height increases.

In Fig. 1 (middle), we show $\partial\left(F_{q} / T\right) / \partial H$ rescaled with the appropriate power of $H$ expected from the $\mathrm{O}(2)$ scaling ansatz and plotted versus $z$. The good scaling behavior suggests that $\partial\left(F_{q} / T\right) / \partial H$ will indeed diverge in the chiral limit and that $H$-dependent contributions to $F_{q} / T$, arising 

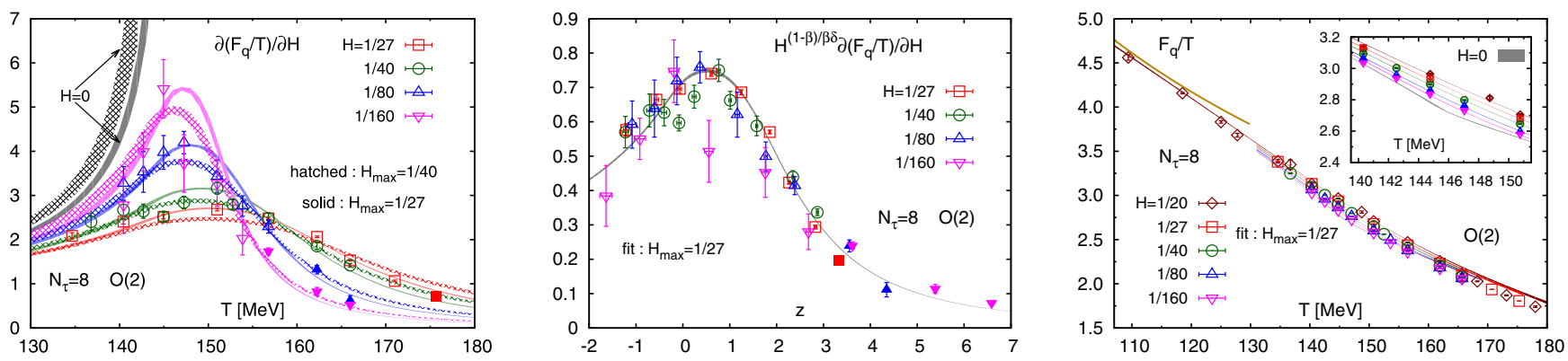

FIG. 1. Derivative of $F_{q}(T, H) / T$ with respect to $H$ as function of $T$ (left) and the rescaled version for the fit with $H_{\text {max }}=1 / 27$ as function of $z$ (middle). Shown are results for several values of $m_{l}$, calculated on lattices with temporal extent $N_{\tau}=8$. Curves in the lefthand figure show results of fits, with and without the data sets for $H=1 / 27$, based on the scaling ansatz given in Eq. (10), with fit parameters given in Table I. Data shown with filled symbols correspond to $z \geq 3$ and have not been included in the fit. The right-hand figure shows $F_{q} / T$ versus $T$. Fits are explained in the text. The inset in the right-hand figure shows data in the temperature range covered by the fits. The chiral limit result for $H=0$ obtained from these fits is shown as gray bands (left, right). The solid gold line in the righthand figure shows the static-light meson contribution to $F_{q} / T$ [23].

from regular terms, are small compared to those coming from the singular part. This motivated a fit ansatz for the regular term that is independent of $H$, i.e., we use $f_{\text {reg }}(T, H=0)$ in all our fits.

A fit to $\partial\left(F_{q} / T\right) / \partial H$ thus only involves the singular term of Eq. (10). We performed this three-parameter fit for all data sets with $H \leq H_{\max }$ by either including or leaving out the data for $H=1 / 27$. These fits are shown in Fig. 1 (left), and the corresponding fit parameters, $A, T_{c}, z_{0}$, are given in Table I. In particular, we find that $T_{c}$, obtained from these fits, as well as the nonuniversal scale parameter $z_{0}$, agree well with earlier fit results for chiral susceptibilities [16] in $(2+1)$-flavor QCD. The rescaled fits are also shown in Fig. 1 (middle).

Data for $F_{q}(T, H) / T$ are shown in Fig. 1 (right). They have been fitted to the scaling ansatz Eq. (5) using only the constant and linear $H$-independent terms in the regular part as fit parameters and keeping fixed the three nonuniversal constants, determined in the previous step, in the singular part. The $T$-range and data included in the fit are shown in the inset. The resulting fit parameters, $a_{0,0}^{r}, a_{1,0}^{r}$, are given in Table I. In this figure, we also show the static-light meson contribution to $F_{q} / T$ calculated in the hadron-gas approximation [23,24]. In chiral perturbation theory, this also gives a linear dependence on $H$ at low temperature [25].

Once we have determined all five fit parameters for $F_{q}(T, H) / T$, we can plug them into Eq. (7) to arrive at a

TABLE I. Summary of fit parameters for $F_{q}(T, H) / T$ and the ratio $R^{+}$introduced in Eq. (13). The parameters for the singular part $\left(A, T_{c}, z_{0}\right)$ have been obtained from a fit to $\partial\left(F_{q} / T\right) / \partial H$.

\begin{tabular}{|c|c|c|c|c|c|c|}
\hline \multicolumn{4}{|c|}{ Singular part } & \multicolumn{2}{|c|}{ Regular part } & \multirow[b]{2}{*}{$R^{+}$} \\
\hline$H_{\max }$ & $A$ & $T_{c}$ & $z_{0}$ & $a_{0,0}^{r}$ & $a_{1,0}^{r}$ & \\
\hline $1 / 27$ & $2.48(2)$ & $145.6(3)$ & $2.24(5)$ & $2.74(1)$ & $-34.4(7)$ & $-0.92(1)$ \\
\hline $1 / 40$ & $2.26(5)$ & $144.2(6)$ & $1.83(9)$ & $2.81(3)$ & $-27(1)$ & $-0.86(1)$ \\
\hline
\end{tabular}

parameter-free description of the $T$ and $H$ dependence of $\langle P\rangle$. The thus determined curves are shown in Fig. 2. As seen in the inset, they agree well with $\langle P\rangle$ data near $T_{c}^{N_{\tau}=8}$, which suggests the behavior of $\langle P\rangle$ is explained well by chiral scaling behavior in this region and serves as a consistency check of our approach.

In Figs. 1 (right) and 2 (top), we also show the chiral limit $(H=0)$ results for $F_{q} / T$ and $\langle P\rangle$. For the former, this is a sum of regular and singular contributions,

$$
\frac{F_{q}(T, 0)}{T}=a_{0,0}^{r}+t\left(a_{1,0}^{r}+A^{ \pm}|t|^{-\alpha}\right),
$$

with $A^{ \pm}=(2-\alpha) z_{0}^{1-\alpha} c_{0}^{ \pm} A$; see Eq. (9). Although at $T_{c}$ the contribution to the slope is entirely given by the regular term $a_{1,0}^{r}$, close to $T_{c}$ this contribution gets to a large extent canceled by the singular contributions, $A^{ \pm}|t|^{-\alpha}$. This is the origin of the well-known spike in specific-heat-like observables (second derivatives with respect to $T$ ) in the $\mathrm{O}(N)$ universality classes. In the chiral limit, our fit results suggest the appearance of such a spike in the temperature derivatives of $F_{q}(T, 0) / T$ as well as $\langle P\rangle$. For the former, we obtain from Eq. (12),

$$
T_{c} \frac{\partial\left(F_{q}(T, 0) / T\right)}{\partial T}=a_{1,0}^{r}\left(1+R^{ \pm}|t|^{-\alpha}\right),
$$

with $R^{ \pm}=(1-\alpha) A^{ \pm} / a_{1,0}^{r} . R^{+}$is given in Table I and $R^{+} / R^{-}=c_{0}^{+} / c_{0}^{-}=1.12(5)$ is a universal ratio [26]. This makes it evident that already for $|t|=0.01$ the slope of $-F_{q} / T$ is about a factor 5 smaller than at $T_{c}$.

The basic features found in our analysis of $(2+1)$-flavor QCD are quite similar to those found in the analysis of $3 d$, $\mathrm{O}(2)$ symmetric spin models [26]. Also in that case a large cancellation of contributions arising from regular and singular terms is found; the spike in the specific heat, $C_{V}$, is concentrated in a temperature interval of about $1 \%$ around $T_{c}$, and $C_{V}$ changes by almost a factor 10 in this 

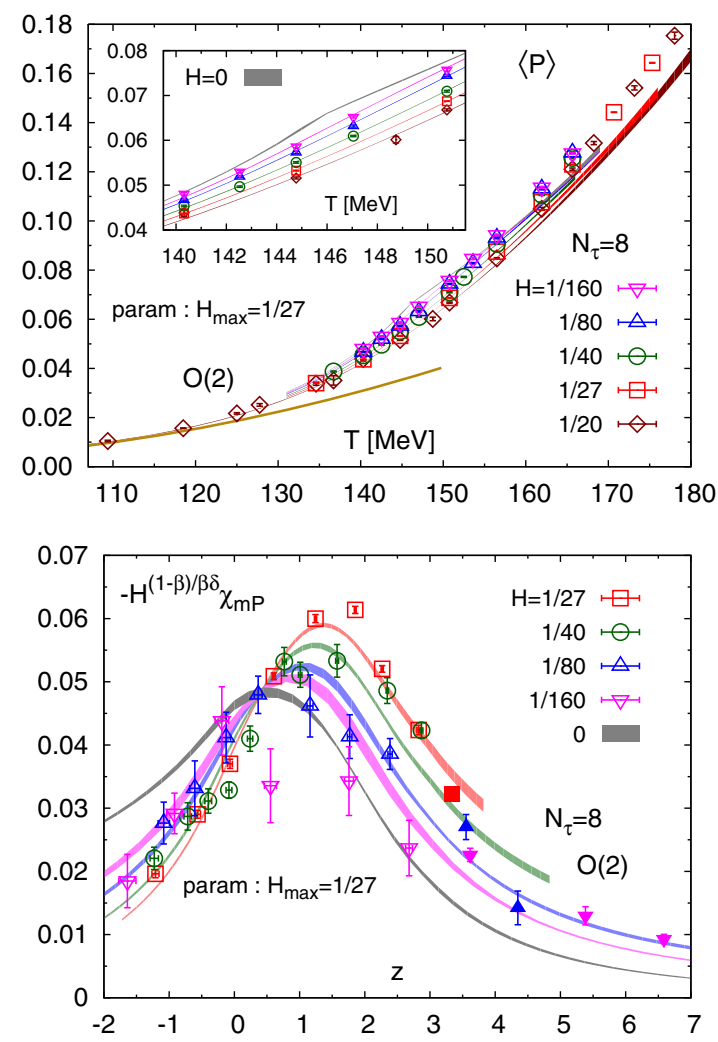

FIG. 2. Top: $T$ dependence of $\langle P\rangle$ obtained on lattices with temporal extent $N_{\tau}=8$ for several values of $H$. The inset shows data in the $T$ range covered by the fit. Curves are based on a fit that used for the singular part parameters for $H_{\max }=1 / 27$ given in Table I. The chiral limit result for $H=0$ obtained from this fit is shown as a gray band. The solid gold line is as described in Fig. 1. Bottom: derivative of $\langle P\rangle$ versus scaling variable $z$.

temperature interval. In Fig. 3, we show a comparison of the scaling behavior of $-T_{c} \partial\left(F_{q}(T, 0) / T\right) / \partial T$, obtained for the case of $(2+1)$-flavor QCD, and $C_{V}$ in $\mathrm{O}(2)$ spin

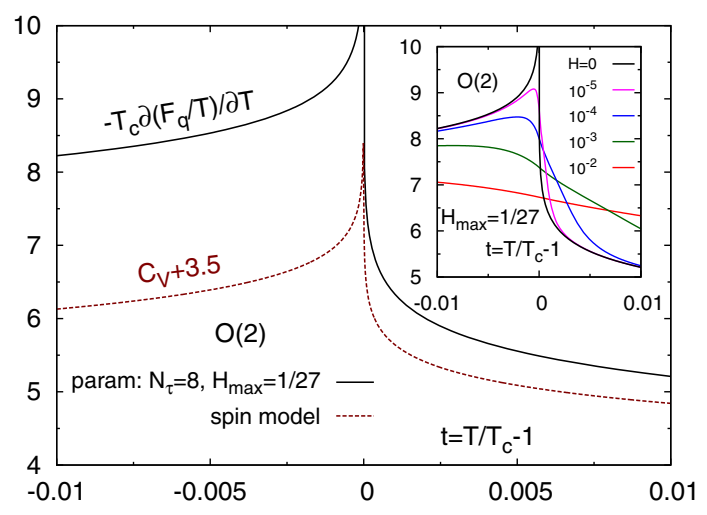

FIG. 3. Comparison of fits to the specific heat at $H=0$ of the $3 d, \mathrm{O}(2)$ spin model taken from Ref. [26] (dashed line) and the negative of the temperature derivative of $F_{q}(T, 0) / T$ (solid line). The former curve is shifted vertically by a constant for easier comparison with the spin model result. models at $H=0$ [26]. The inset shows the development of this sharp peak as the quark mass decreases. It makes clear that this feature becomes visible only for $H$ being substantially smaller than the region $H \simeq 10^{-2}$ that is accessible in current lattice QCD calculations.

\section{CONCLUSIONS}

We have examined the quark mass dependence of the Polyakov loop expectation value and the heavy quark free energy extracted from it. We provided evidence for the influence of chiral symmetry restoration that manifests in the singular behavior of the quark mass derivatives of $\langle P\rangle$ and $F_{q} / T$ and arises from the energylike behavior of these observables with respect to chiral transformations. These derivatives diverge at $T_{c}$ in the chiral limit, consistent with the expected behavior for energylike observables in the $3 d$, $\mathrm{O}(2)$ universality class.

We showed that at finite values of the lattice spacing the relative distribution between singular and regular contributions to the energylike variables in QCD seems to be similar to that in the $3 d, \mathrm{O}(2)$ spin model. In particular, in the chiral limit, the very narrow spike showing up in specific-heat-like observables, which results from a partial cancellation of singular and regular contributions combined with the existence of a quite small, negative critical exponent $\alpha$, is expected to show up also in the $T$-derivatives of $F_{q} / T$ and $\langle P\rangle$.

As the critical exponent $\alpha$ also is negative and small in the $\mathrm{O}(4)$ universality class, similar behavior of $F_{q} / T$ and $\langle P\rangle$ is expected to persist in the continuum limit. However, as $|\alpha|$ is an order of magnitude larger compared to the $\mathrm{O}(2)$ case, the spike may become broader and may also be more prominent already for larger quark masses. This may be tested also in effective model calculations.

All data from our calculations, presented in the figures of this paper, can be found in [27].

\section{ACKNOWLEDGMENTS}

This work was supported by the Deutsche Forschungsgemeinschaft (DFG, German Research Foundation) Project No. 315477589-TRR 211 and by the German Bundesministerium für Bildung und Forschung through Grant No. 05P18PBCA1. Numerical calculations were made possible through PRACE grants at CSCS, Switzerland and grants at the Gauss Centre for Supercomputing and NIC-Jülich, Germany. These grants provided access to resources on Piz Daint at CSCS as well as on JUQUEEN and JUWELS at NIC. This research also used awards of computer time at NERSC, Berkeley Laboratory, provided by the DOE ASCR Leadership Computing Challenge program. We thank the HotQCD Collaboration for providing access to their latest data sets and for many fruitful discussions. 
[1] L. G. Yaffe and B. Svetitsky, Phys. Rev. D 26, 963(R) (1982).

[2] L. D. McLerran and B. Svetitsky, Phys. Lett. B 98, 195 (1981).

[3] J. Kuti, J. Polonyi, and K. Szlachanyi, Phys. Lett. B 98, 199 (1981).

[4] Y. Aoki, S. Borsanyi, S. Durr, Z. Fodor, S. D. Katz, S. Krieg, and K. K. Szabo, J. High Energy Phys. 06 (2009) 088.

[5] S. Borsanyi, Z. Fodor, C. Hoelbling, S. D. Katz, S. Krieg, C. Ratti, and K. K. Szabo (Wuppertal-Budapest Collaboration), J. High Energy Phys. 09 (2010) 073.

[6] A. Bazavov, N. Brambilla, H. T. Ding, P. Petreczky, H. P. Schadler, A. Vairo, and J. H. Weber, Phys. Rev. D 93, 114502 (2016).

[7] D. A. Clarke, O. Kaczmarek, F. Karsch, and A. Lahiri, Proc. Sci., LATTICE2019 (2020) 194.

[8] R. D. Pisarski and F. Wilczek, Phys. Rev. D 29, 338 (1984).

[9] L. D. McLerran and B. Svetitsky, Phys. Rev. D 24, 450 (1981).

[10] K. G. Wilson, Phys. Rev. B 4, 3174 (1971).

[11] K. G. Wilson, Phys. Rev. B 4, 3184 (1971).

[12] J. Engels and F. Karsch, Phys. Rev. D 85, 094506 (2012).

[13] J. Engels, S. Holtmann, T. Mendes, and T. Schulze, Phys. Lett. B 492, 219 (2000).

[14] M. Hasenbusch and T. Török, J. Phys. A 32, 6361 (1999).

[15] D. A. Clarke, O. Kaczmarek, A. Lahiri, and M. Sarkar, arXiv:2010.15825.

[16] H. T. Ding, P. Hegde, O. Kaczmarek, F. Karsch, A. Lahiri, S.-T. Li et al., Phys. Rev. Lett. 123, 062002 (2019).

[17] A. Bazavov, T. Bhattacharya, M. Cheng, C. DeTar, H. T. Ding, S. Gottlieb et al., Phys. Rev. D 85, 054503 (2012).
[18] A. Bazavov, T. Bhattacharya, C. DeTar, H. T. Ding, S. Gottlieb, R. Gupta et al. (HotQCD Collaboration), Phys. Rev. D 90, 094503 (2014).

[19] A. Bazavov, H. T. Ding, P. Hegde, O. Kaczmarek, F. Karsch, E. Laermann et al., Phys. Rev. D 95, 054504 (2017).

[20] E. Follana, Q. Mason, C. Davies, K. Hornbostel, G. P. Lepage, J. Shigemitsu, H. Trottier, and K. Wong (HPQCD, UKQCD Collaborations), Phys. Rev. D 75, 054502 (2007).

[21] A. Bazavov, H. T. Ding, P. Hegde, O. Kaczmarek, F. Karsch, N. Karthik et al. (HotQCD Collaboration), Phys. Lett. B 795, 15 (2019).

[22] A. Bazavov, C. Bernard, C. DeTar, X. Du, W. Freeman, S. Gottlieb et al. (MILC Collaboration), Proc. Sci., LATTICE2010 (2010) 074.

[23] A. Bazavov and P. Petreczky, Phys. Rev. D 87, 094505 (2013).

[24] E. Megias, E. R. Arriola, and L. L. Salcedo, Phys. Rev. Lett. 109, 151601 (2012).

[25] N. Brambilla, J. Komijani, A. S. Kronfeld, and A. Vairo (TUMQCD Collaboration), Phys. Rev. D 97, 034503 (2018).

[26] A. Cucchieri, J. Engels, S. Holtmann, T. Mendes, and T. Schulze, J. Phys. A 35, 6517 (2002).

[27] D. A. Clarke, O. Kaczmarek, F. Karsch, A. Lahiri, and M. Sarkar, https://doi.org/10.4119/unibi/2950112.

Correction: A minor error in the first sentence of the Abstract has been fixed. 